\title{
SUKIT
}

\section{Combining Cloud and Grid with a User Interface}

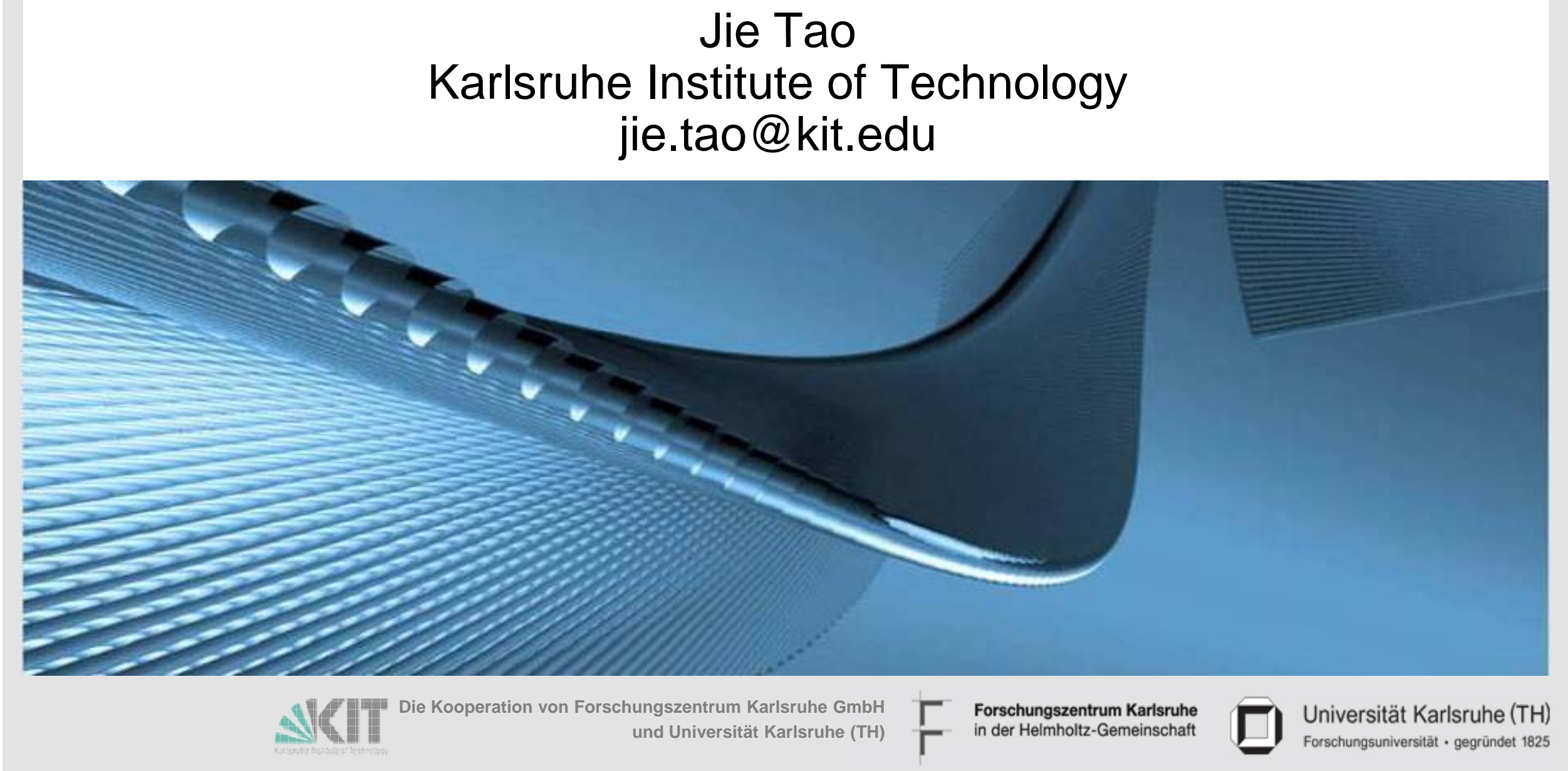




\section{Outline}

- Motivation

- The g-Eclipse Project

- Extending g-Eclipse for a Cloud Framework

- Initial Implementation: Accessing the Amazon Web Service

- Conclusion 


\section{Motivation}

- Various clouds co-exist

$\square$ Amazon EC2, S3

$\square$ Google App Engine

$\square$ Microsoft Azure

$\square$ Eucalyptus, Cumulus, Nimbus, OpenNybula

- Different user interfaces (GUI or command-line)

$\square$ Working with several clouds: must know each interface

$\square$ Interaction between clouds: not easy

- Moving grid to cloud

$\square$ Running grid jobs on the cloud

$\square$ Grid as a Service (RightScale)

- Goal: developing a generic, intuitive cloud user interface 


\section{g-Eclipse: A General Framework for Accessing the Grid}

- Many application domains start using Grid infrastructures

- But...

$\square$ Grid technology is complex

- Different systems are used

- Middleware (Unicore, gLite, Globus, GRIA, ...)

- Many separate tools (i.e for installation, monitoring, ...)

$\square$ Different programming paradigms

- Batch type systems vs. service oriented systems

- Many programming languages

- e-Users want to interact with the grid infrastructure

$\square$ Without knowing all details (development, deployment, testing, management, ...)

- $\rightarrow$ Tooling is necessary!!

$\square$ Wizards, Editors, ...

$\square$ Hide the complexity!! 


\section{g-Eclipse: the Idea}

- Provides a general UI framework/eco system

$\square$ Designed for application users, resource providers, and application developers

$\square$ Middleware independent core infrastructure + middleware extension

$\square$ Allowing users to access the grid in a simple way

- File transfer: drag\&drop

- Job submission: mouse click

- (Re-)use Eclipse and contribute

$\square$ Eclipse is an eco system

- Build for extension

- More than a JAVA IDE

- The biggest "coordinated" Open Source project

$\square$ Gain OS independence (by using JAVA) 


\section{g-Eclipse: grid user roles \& use cases}

- 3 different roles

$\square$ User

$\square$ Operator

$\square$ Developer

- In general...

$\square$ Job management

$\square$ Resource management

- Files

- Applications

- Hardware

$\square$ Application deployment

$\square$ Infrastructure monitoring

$\square$ Application development

$\square$ Visualization tools

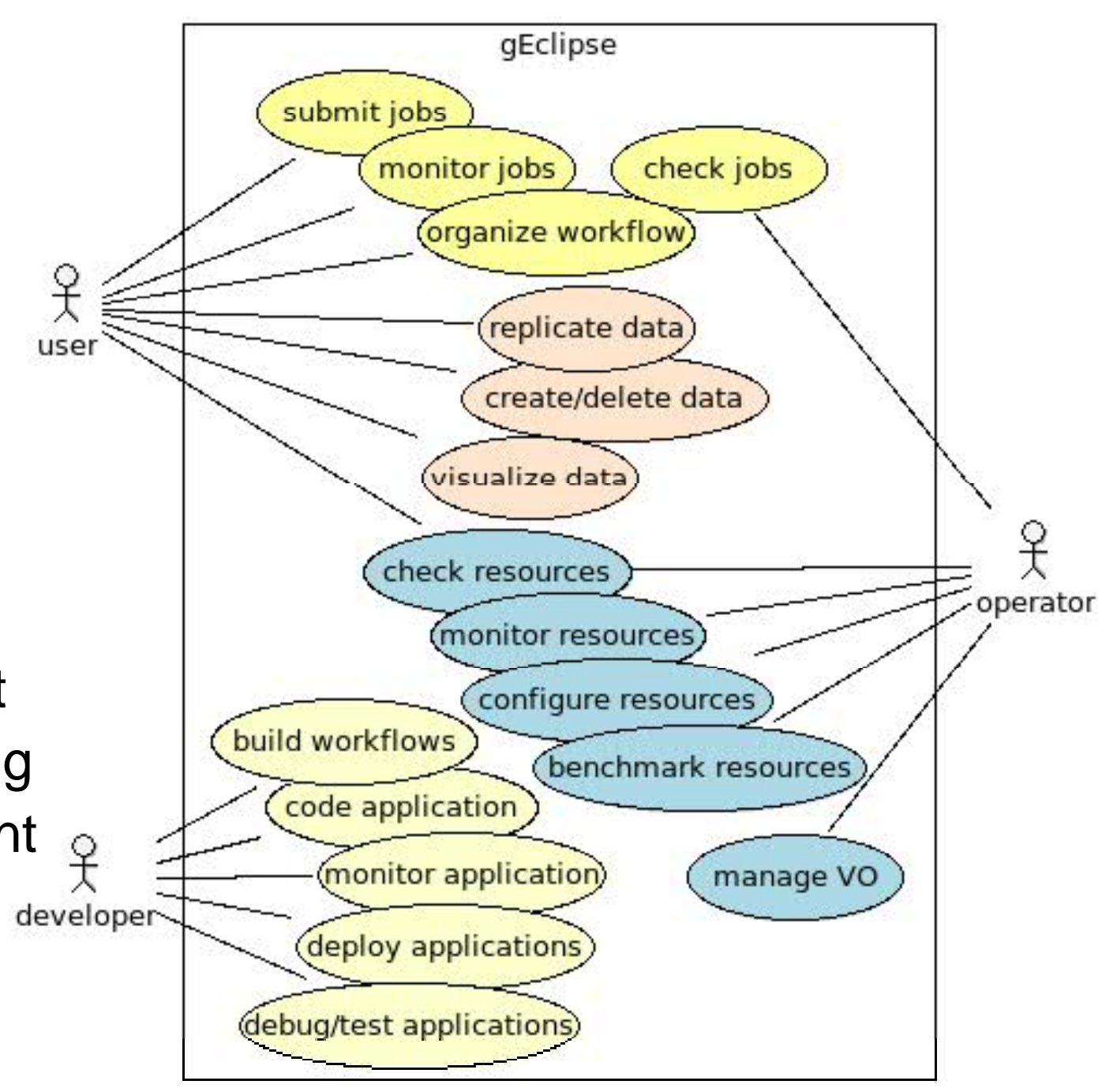




\section{g-Eclipse: screenshot}

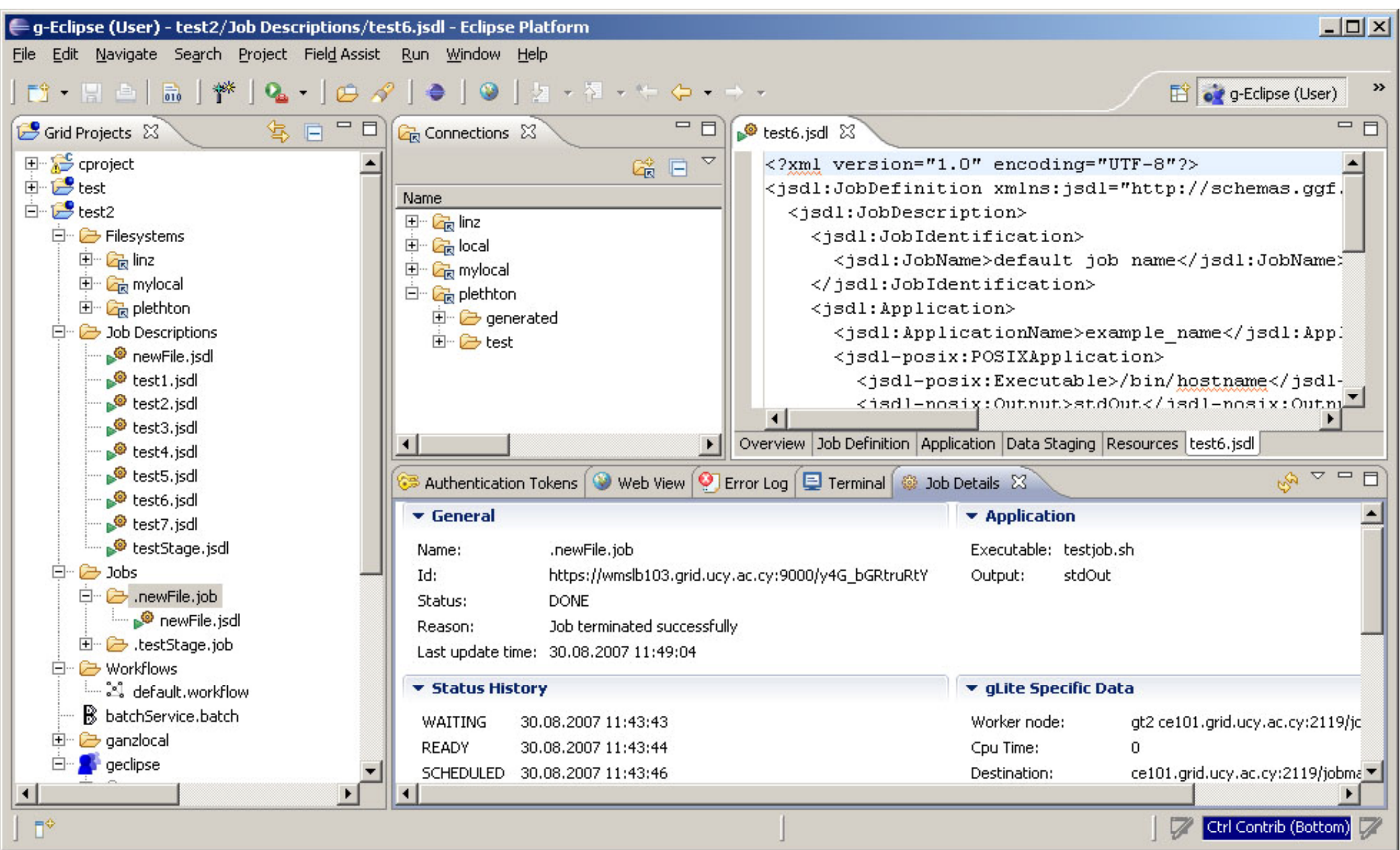




\section{g-Eclipse: architecture overview}

- Abstraction Layer

$\square$ Core functionalities, e.g.

- Authentication/Authorization

- VO management

- Data management

- Job submission

$\square$ Common user interface, e.g.

- Views

- Wizards

- Dialogs

- Preference pages

- Implementation Layer

$\square$ Extended core functionalities

$\square$ Middleware specific functionalities

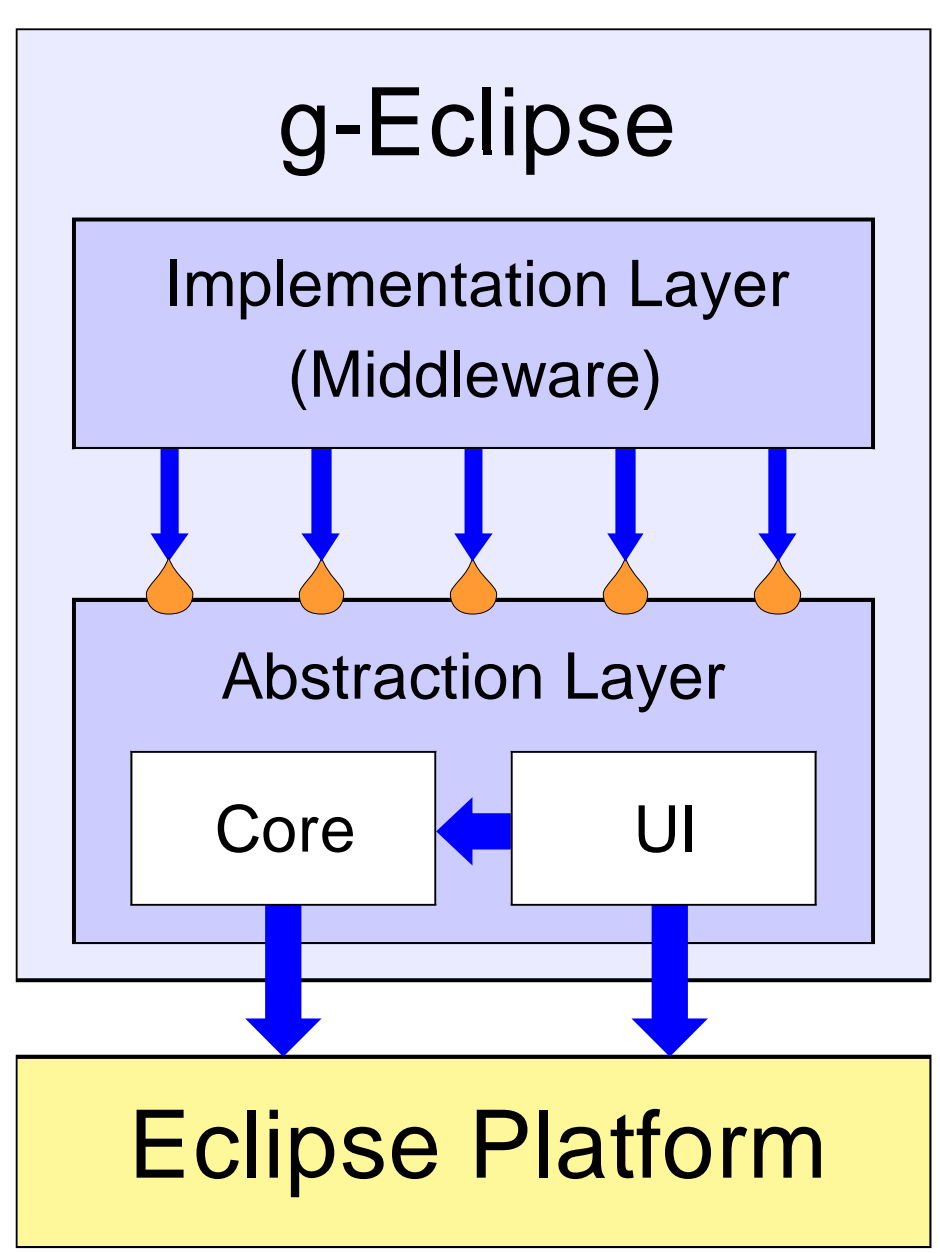

Eclipse Extension Point 


\section{Extending g-Eclipse for Clouds}

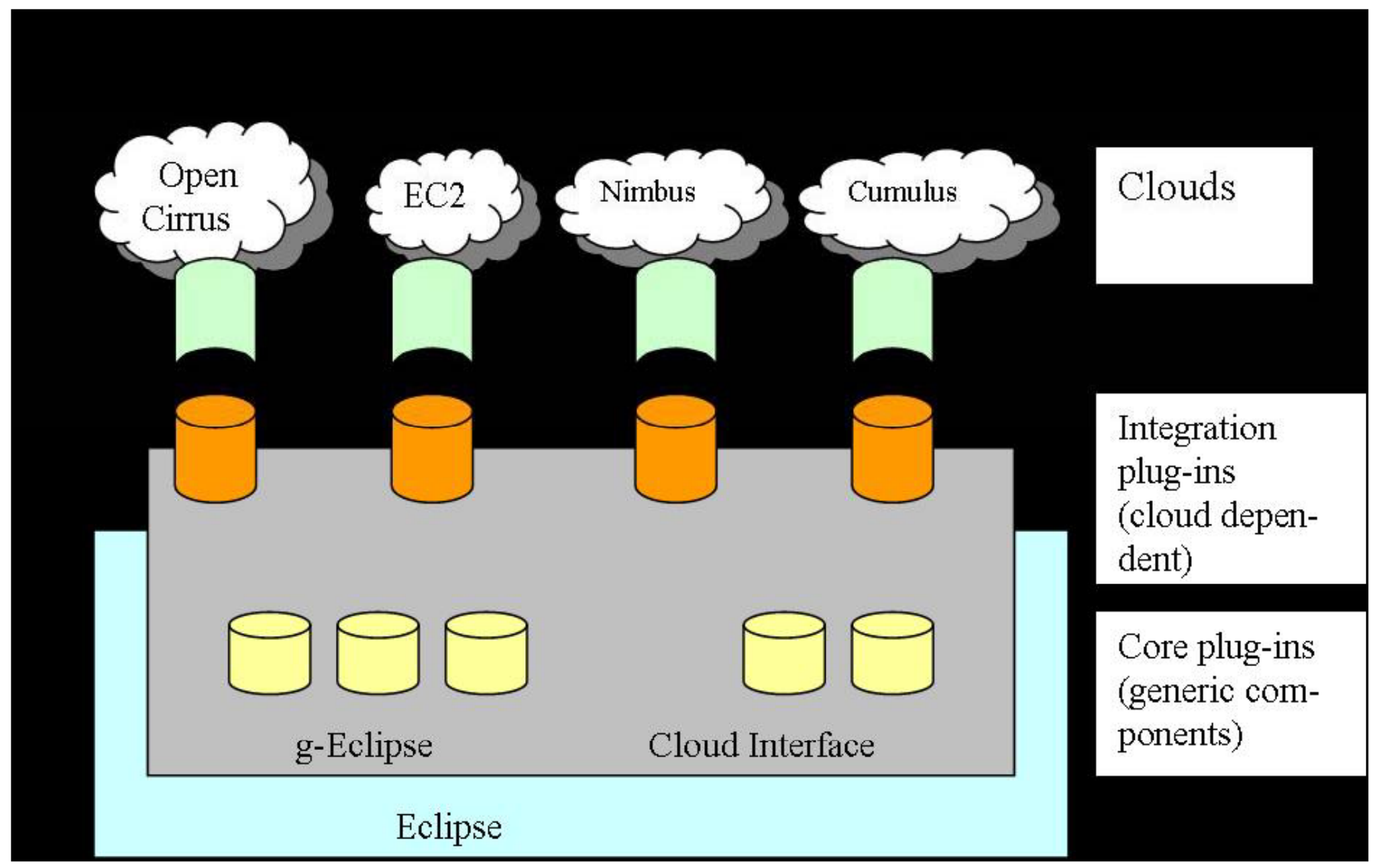




\section{Extending g-Eclipse for Clouds (cont.)}

- An additional folder: service description

$\square$ g-Eclipse core

- Common interfaces for cloud service

- Extension of VO management

$\square$ g-Eclipse UI:

- Multi-layer editor for service specification, supporting

$\square$ HaaS, SaaS, HPC as a Service, Storage as a Service, Grid as a Service

- Actions: service request, service execution, service termination

- Views: available services, attributes, ...

- Templates for application development (Map/Reduce, web service...)

- Middleware extension

$\square$ Specific implementation for individual clouds

- Wizards and views for authentication token, service deployment/execution/termination, .... 


\section{Initial Implementation: Accessing the Amazon}

Web Service (I)

- AWS VO implementation

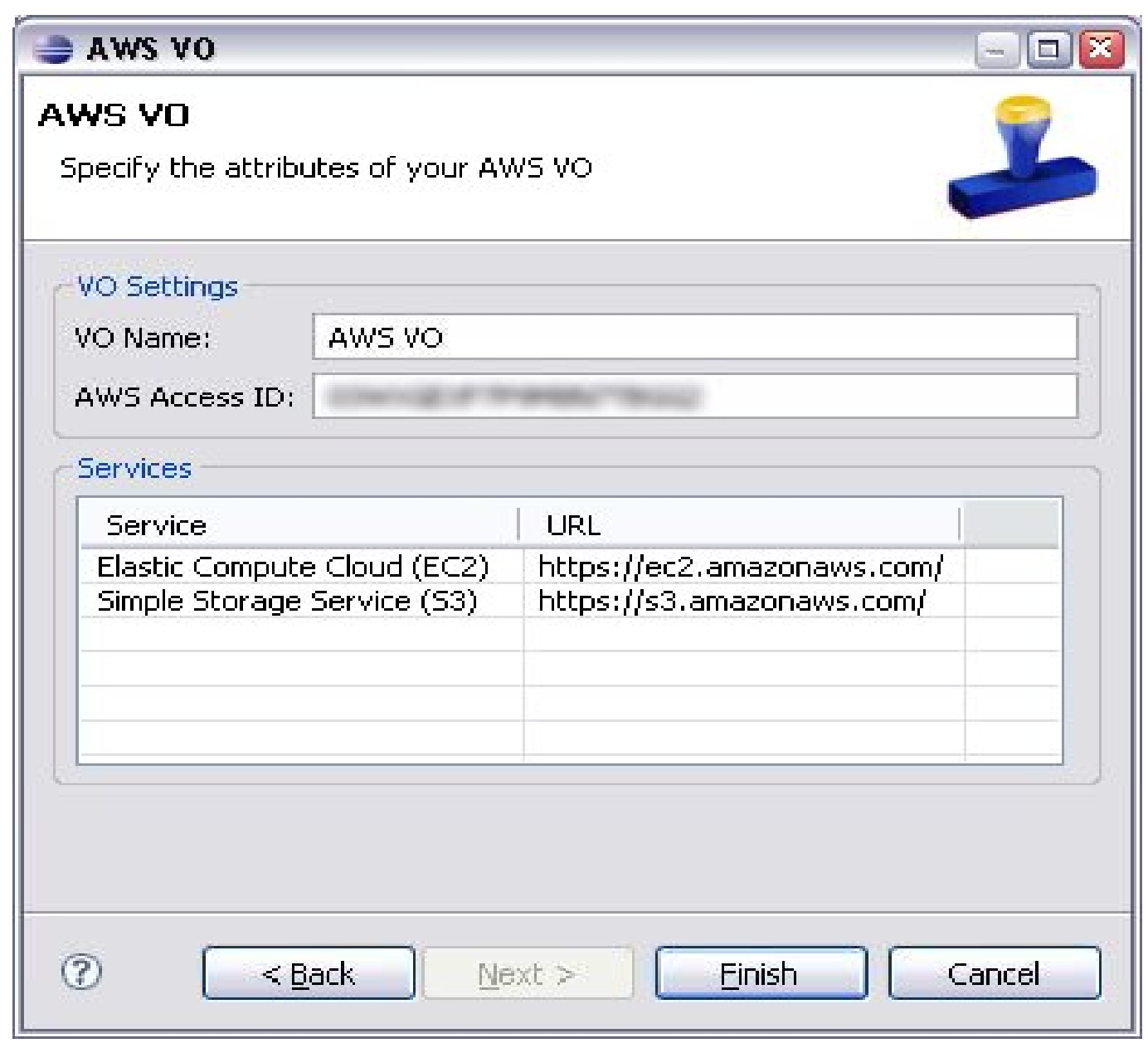




\section{Initial Implementation: Accessing the Amazon}

Web Service (II)

- Authentication token for AWS credential

\begin{tabular}{|l|l||}
\hline Create new authentication token \\
Provide authentication credentials \\
\hline Amazon web Service Credentials \\
Access ID: \\
Secret ID: \\
\hline \\
\hline (3) Back \\
\hline
\end{tabular}




\section{Initial Implementation: Accessing the Amazon Web Service (III)}

- Project view

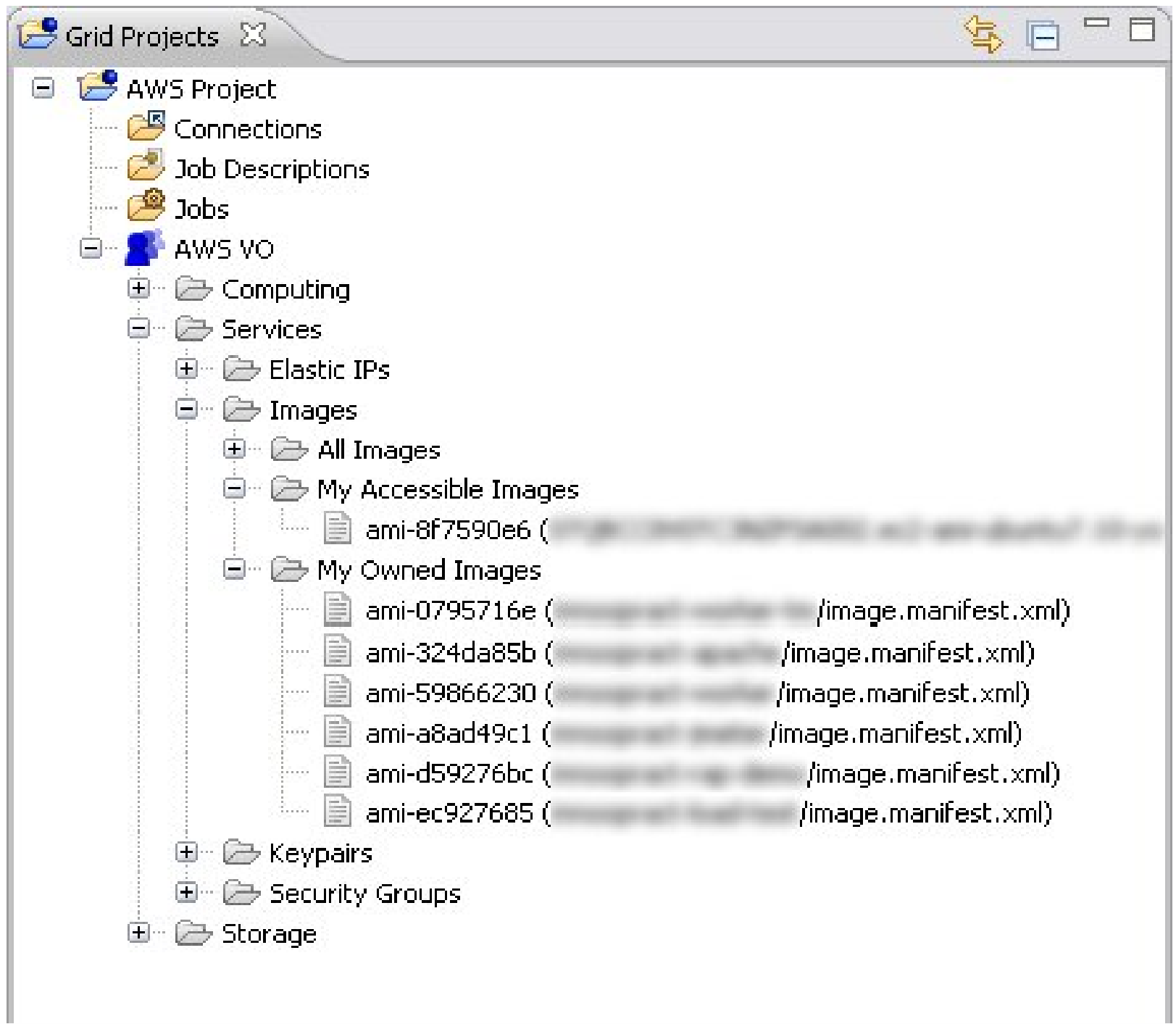




\section{Initial Implementation: Accessing the Amazon Web Service (IV)}

- AMI properties

\begin{tabular}{|c|c|c|}
\hline Properties \&3 & & 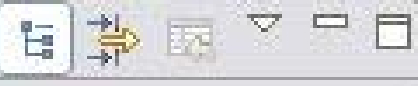 \\
\hline Property & Value & \\
\hline \multicolumn{3}{|l|}{$\exists$ General } \\
\hline Availability Zone & us-east-1a & \\
\hline DNS Name & ec2-75-101-226-103.compute-1. amazonaws.com & \\
\hline Image Id & ami-1454b17d & \\
\hline Instance Id & $\mathrm{i}-98 \mathrm{fc} 37 \mathrm{f} 1$ & \\
\hline Instance Type & m1.small & \\
\hline Kernel Id & $a k j-a 71 c f 9 c e$ & \\
\hline Key Name & innoopract-keypair & \\
\hline Launch Time & $04.06 .200810: 52: 46$ CEST & \\
\hline Private DNS Name & ip-10-251-215-48,ec2, internal & \\
\hline Ramdisk Id & ari-a51cf9cc & \\
\hline \multicolumn{3}{|l|}{ Reason } \\
\hline State & running (16) & \\
\hline
\end{tabular}




\section{Initial Implementation: Accessing the Amazon}

Web Service (V)

- Service execution: launching an AMI

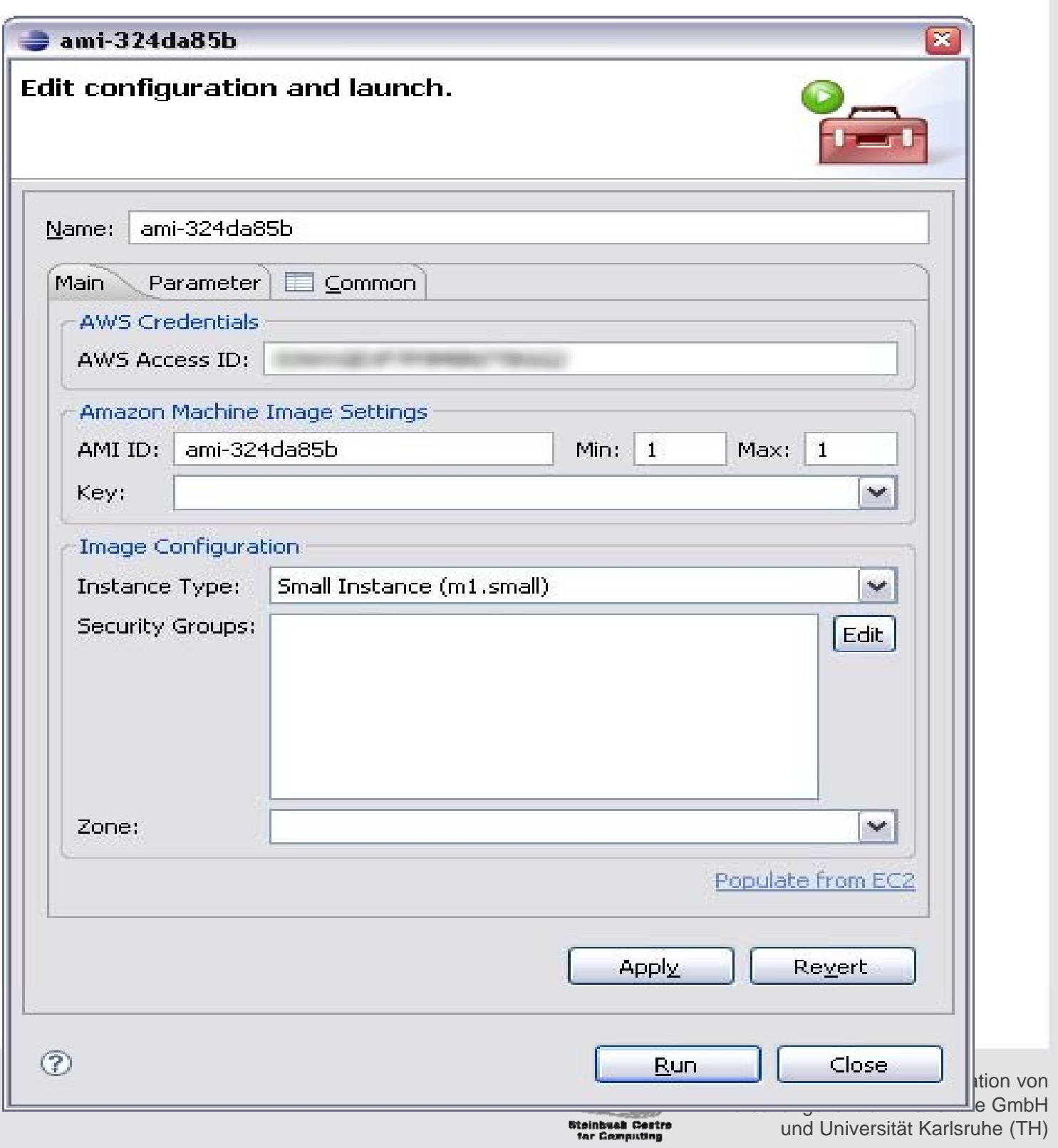




\section{Initial Implementation: Accessing the Amazon}

Web Service (IV)

- Remote login
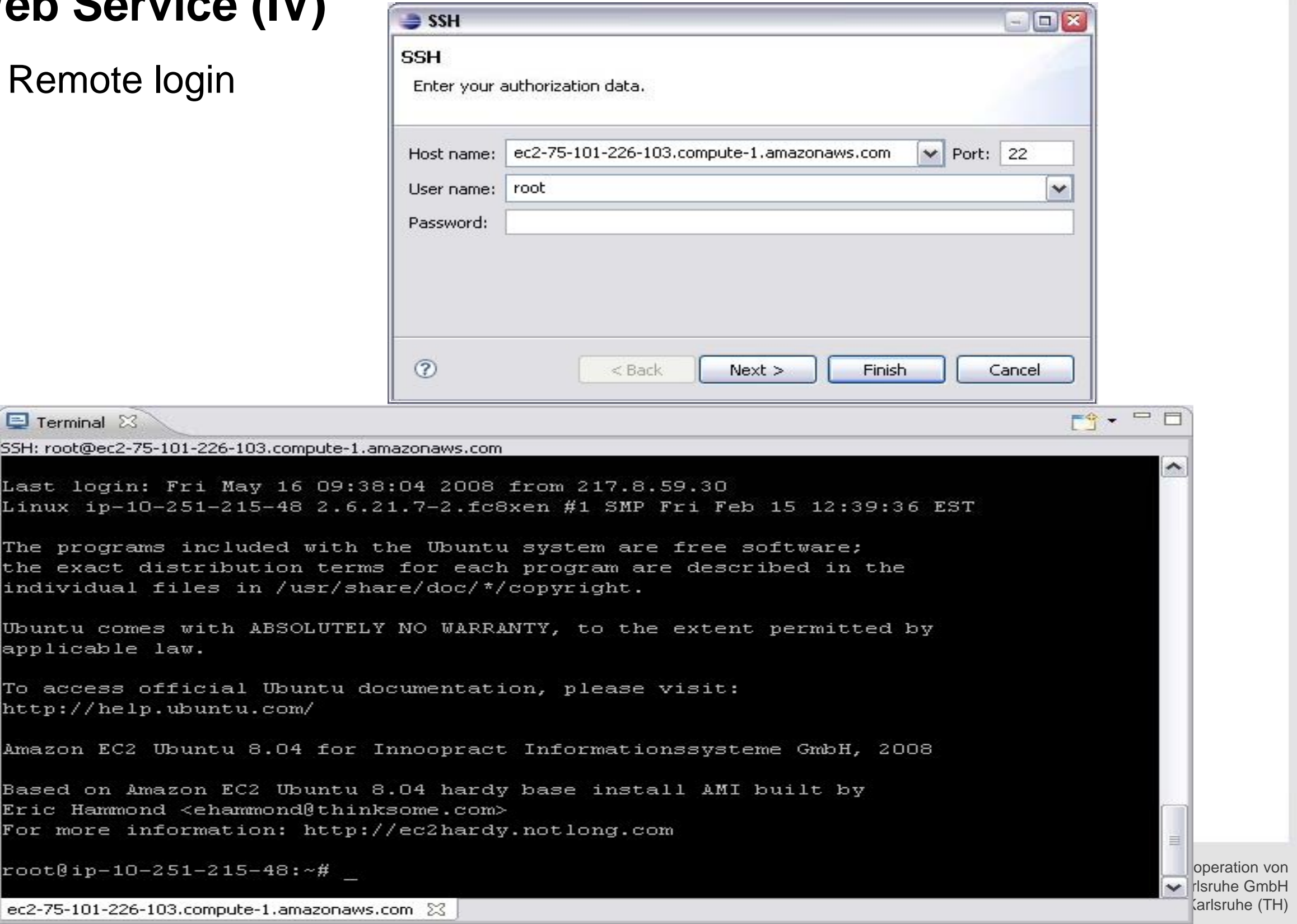


\section{Conclusion}

- $g$-Eclipse is a generic framework for simplifying the access to the grids

- Extending g-Eclipse for accessing the clouds

$\square$ An easy way to interact with computing clouds

$\square$ A bridge across clouds and grids

- Core extensions and specific implementation for AWS and Eucalyptus

- Further work

$\square$ Full functionality of the cloud user interface

$\square$ Connecting more clouds 